\title{
Improving the Performance of Modular Production in the Apparel Assembly: A Mathematical Programming Approach
}

\author{
Xiaoqing Wang, ${ }^{1}$ Chun-Hung Chiu, ${ }^{2}$ and Wei Guo ${ }^{3}$ \\ ${ }^{1}$ Lingnan (University) College, Sun Yat-Sen University, Guangzhou 510275, China \\ ${ }^{2}$ Sun Yat-Sen Business School, Sun Yat-Sen University, Guangzhou 510275, China \\ ${ }^{3}$ Guangdong Hydropower Planning \& Design Institute, Guangzhou 510635, China
}

Correspondence should be addressed to Xiaoqing Wang; xqwang2@gmail.com

Received 5 April 2014; Revised 10 June 2014; Accepted 17 June 2014; Published 3 July 2014

Academic Editor: Tsan-Ming Choi

Copyright (c) 2014 Xiaoqing Wang et al. This is an open access article distributed under the Creative Commons Attribution License, which permits unrestricted use, distribution, and reproduction in any medium, provided the original work is properly cited.

\begin{abstract}
We construct the mathematical models to find the optimal allocation of the modules capacity (module members) to different assembly operations in a module for given garment assembly tasks in a modular production system. The objectives of the models are minimizing the holding cost for work in process (WIP) inventories in the module and the total deviation of the WIP inventories from their corresponding target values in the module during a specific time interval. The solutions of the models can be used as reference to achieve better allocation of the module members to different operations in a module to fulfill the given garment assembly tasks.
\end{abstract}

\section{Introduction}

In the apparel market, there are tremendous different types of products, the corresponding demand for each type of product is volatile and unpredictable, and the life cycle of the products is very short. The apparel retailers need to respond quickly to the changing market in order to keep their survivals $[1,2]$. Consequently, the retailers require their suppliers to replenish the products quickly with the exact amount they want [3-8].

On the other hand, the apparel assembly in the traditional apparel manufacturing is very time-consuming $[9,10]$. The production system used for apparel assembly is the progressive bundle system (PBS). In PBS, each operation is done by a single worker who operates a stationary machine. The worker receives the unfinished garments in bundles; he (or she) then performs the single operation on each garment. The garments then move to the next worker for the next operation in bundles. The main philosophy behind PBS is maximizing the utilization of each worker's working capacity. Due to the different and uncertain operating time needed for different operations in given assembly tasks [11-13], PBS has to depend heavily on the buffers between operations to avoid the starvation of garments at some operations. Thus, in PBS, the throughput time (from the cutting pieces to finished garments) is long and work in process (WIP) inventory is high $[3,9]$.

To meet the retailers' requirements and, at the same time, keep their own inventory as low as possible, the apparel manufacturers need to employ the more flexible production systems for the apparel assembly [14]. Although the assembly process in PBS can be modified to achieve some degree of flexibility $[15,16]$, employing modular production system (MPS) [3, 17-21] with some performance improvement strategy [5] is more promising to achieve these goals. In MPS, the module, which consists of a group of workers, is in charge of a part of, sometimes a whole, garment. In most of the time, each module member performs a single assembly task, just like in PBS. But if WIP inventory is building up at some operations, the module members at other operations will move to those operations to help, thus increasing the work capacity and decreasing the inventory at those operations. So, in MPS, the throughput time is short and WIP inventory is 
small $[22,23]$. It seems that MPS is the perfect solution for the apparel manufacturer to respond to the quick replenishment requirement by the retailers.

Although MPS can provide obvious benefits for the apparel manufactures, the diffusion of MPS in the apparel industry is slow. The possible reasons for this phenomenon are studied by the researchers, most from the perspective of human resource practices $[20,21]$.

In this paper, we focus on the implementation of MPS on the operational level. Based on a novel mathematical programming model called separated continuous linear programming (SCLP) and its extension, separated continuous conic programming (SCCP), we construct two mathematical models to find the optimal allocation of the module's capacity (module members) to different operations for given assembly tasks. The objectives of the models are minimizing the holding cost for WIP inventories in the module and the total deviation of the WIP inventories from their corresponding target values in the module during a specific time interval. The solutions of the models can be used as reference to achieve better allocation of the module members to operations in the module so that the building-up of bottleneck in the apparel assembly can be avoided and the flow balance can be achieved.

To our knowledge, our work is the first one to facilitate the adoption of MPS by addressing the optimal allocation of module members to operations for given assembly tasks.

The paper is organized as follows. In the next section, the literature on the adoption and performance of MPS in the apparel industry is reviewed. We also give a brief introduction to SCLP and SCCP on which our models are based. In Section 3, we present two models for optimal allocation of the module members to different operations for given assembly tasks in a module to achieve the minimization of the holding cost of WIP inventories and the total deviation of the WIP inventories from their corresponding target values during a specific time interval. In Section 4 we summarize what we get and point out some future research direction.

\section{Literature Review}

2.1. Literature on the Adoption and Performance of MPS in the Apparel Manufacturing. As we said before, MPS allows apparel manufacturers to respond quickly to the replenishment requirement of the retailers and, at the same time, reduce their own need to hold large WIP inventory. Even more, there is also research showing that the workers in the MPS earn more than their counterparts in the PBS [24]. But still the adoption level of MPS in apparel manufacturing is low $[20,21]$.

Research shows that there are several key factors affecting the performance of MPS in a specific apparel manufacturer and, consequently, affecting the adoption level of MPS in that apparel manufacturer. The most important factor is the human resource practices.

By using the empirical study, [20, 21] show that the proper modification of human resources practices from PBS to MPS is crucial for the smooth transition from PBS to
MPS in many apparel manufacturers. In PBS, the objective is maximizing the individual worker's output. The sewing process is highly fragmented and each worker undertakes one operation in isolation. The communication between workers is minimized. On the contrary, in MPS, the objective is maximizing the output of each module. Each module member undertakes one or more operations. The operations each module member performs as well as the order in which they perform those operations are different for different assembly tasks. The interaction among module members is very important to achieve the continuous balance of the flow of production and the module's goal. The modification of human resource practices is necessary to reflect these differences. For example, the incentive system in MPS should focus on group incentive in order to encourage the module members to communicate, coordinate, and cooperate to maximize the module's output. The training arrangement should be also modified to encourage module members to learn more operations from training and from other module members. The supervision should be modified too. The supervisors should consider the functions each module member plays and how they become involved in the module work. The importance of the modification of human resource practice is also observed in a case study by using simulation method [25].

The modifications of human resource practices pose a huge work for the apparel manufacturers who want to adopt to MPS, and the results of those modifications affect a lot the throughput and replenishment speed the manufacturer could achieve.

Apart from the human resource practices, research also identifies other factors affecting the adoption and performance of MPS in the apparel manufacturing, including the timing of adoption, the relationship between the apparel manufacturer and its retailers, the information system used in the apparel manufacturer and retailers, the level of cooperation within the apparel manufacturer, and the completeness of the adoption plan.

$\mathrm{Xu}$ et al. [26] showed that when market is quite volatile, it is optimal for the manufacturer to postpone adoption of MPS. Forza and Vinelli [27] showed that the close collaboration between retailers and manufactures is critical for MPS adoption due to the fact that the availability of current sales data in real time allows the apparel manufacturer to know which product is selling better and therefore to understand market trends in a more comprehensive way. Of course, the information system in the manufacturer should be able to process these data so that more accurate demand information can be obtained. Also, the short throughput time provided by MPS makes no sense if the apparel manufacturers cannot ship their products efficiently to where the retailers require [20]. The adoption plan should consider all the factors mentioned above before starting the adoption process.

There is still other research on the adoption of MPS from different perspective. For example, Lin et al. [28] point out that the production systems used by apparel manufacturers should be significantly related to their manufacturing strategy. The adoption of MPS is proper only when MPS matches the manufacturing strategy of the apparel manufacturers. 
Moreover, besides MPS, there are other studies and models on the production in fashion apparel. For example, Tomastik et al. [29] develop an accurate and low-order integer programming model which integrates scheduling and resource allocation for garments production; Rose and Shier [30] apply an exact enumerative approach for the cloth cut scheduling problem; Hsu et al. [31] formulate a mixed integer programming model for the scheduling problem for yarn-dyed textile manufacturing and propose a genetic algorithm to solve the problem; Yeung et al. [32, 33] study the supply chain coordination and scheduling problem under different settings, and fast algorithms are developed to solve the complex problem; and Choi et al. [34] investigate the scheduling and coordination problem of a multisuppliers, single-warehouseoperator single manufacturer supply chain. They formulate the problem as a two-machine common-due-window flow shop problem and develop two algorithms to solve the optimal scheduling and coordination problems.

In this paper, we focus on the implementation of MPS in the operational level. Specifically, we address the optimal allocation of the module members to different operations for given assembly tasks so that the specific performance of interested can be improved.

We are unaware of any paper focusing on the implementation of MPS in the operational level. The only related paper we found is that of Guo et al. [10]. In that paper, a mathematical model of the job shop scheduling (JSS) problem for PBS is proposed. The objective is minimizing the total penalties of earliness and tardiness of order fulfillment by deciding when to start each order's production and how to assign the operations to module members.

In the next subsection, we will give a brief introduction to SCLP and SCCP, based on which our models are built.

\subsection{Brief Introduction to SCLP and SCCP. Linear program-} ming (LP) is a mathematical model which has extremely wide range of industrial applications. It can be solved fast even with very large size [35]. For this reason, LP has been pushed and extended to an ever-broadening frontier. SCLP is one of such frontier extensions which was first introduced by Anderson [36] who used it to model the job shop scheduling problem.

There are several forms of SCLP. The following is due to Weiss [37]:

$$
\begin{aligned}
& (\mathrm{SCLP}) \quad \max \int_{0}^{T}\left[(\gamma+(T-t) c)^{\prime} u(t)+d^{\prime} x(t)\right] d t \\
& \text { s.t. } \quad \int_{0}^{t} G u(s) d s+F x(t) \leq \alpha+t a, \\
& H u(t) \leq b, \\
& u(t) \geq 0, \quad x(t) \geq 0, \quad t \in[0, T],
\end{aligned}
$$

where $u(t), x(t)$ are decision variables and are assumed to be bounded measurable functions with the measure of the break-point set being $0 . \gamma, c, d, \alpha, a$, and $b$ are vectors; $G, F$, and $H$ are matrices. ' denotes the transpose operation. The word "separated" refers to the fact that there are two kinds of constraints in SCLP: the constraints involving integration and the instantaneous constraints [38].

There exists a rich literature on duality theory and algorithms for SCLP. Interested reader may refer to [39] for the detailed review on the research results on SCLP. Specifically, Wang et al. [40] suggested a polynomial approximation algorithm for SCLP which produces a solution with any predefined precision. The resulted control $u(t)$ is a piecewise constant function of time (bang-bang control) which can be implemented conveniently in practice. In that paper, they further extend SCLP to SCCP which has the following form:

$$
\begin{aligned}
(\mathrm{SCCP}) \quad \max & \int_{0}^{T}\left[(\gamma+(T-t) c)^{\prime} u(t)+d^{\prime} x(t)\right] d t \\
\text { s.t. } \quad & \alpha+t a-\int_{0}^{t} G u(s) d s-F x(t) \in \mathscr{K}_{1}, \\
& b-H u(t) \in \mathscr{K}_{2}, \\
& u(t) \in \mathscr{K}_{3}, \quad x(t) \in \mathscr{K}_{4}, \quad t \in[0, T],
\end{aligned}
$$

where $\mathscr{K}_{i}, i=1,2,3,4$, are closed convex cones in the Euclidean space with appropriate dimensions.

Remark. SCCP (2) can also be regarded as an extension for conic programming $(\mathrm{CP})$ which has the following form:

$$
\begin{array}{ll}
\text { (CP) } & \min \quad c^{\prime} x \\
& \text { s.t. } A x-b \in \mathscr{K},
\end{array}
$$

where $A$ is an $m \times n$ matrix, $b \in \mathfrak{R}^{m}, c \in \mathfrak{R}^{n}$, and $\mathscr{K}$ is a closed convex cone in $\mathfrak{R}^{m}$. Interested reader can refer to Nesterov and Nemirovskii [41] for the detailed information on CP.

Wang et al. [40] also proposed a polynomial approximation algorithm for SCCP which produces a solution with any predefined precision. The resulted solution is also bang-bang. Because of these results, SCLP and SCCP are particularly useful to model large-size problems, usually the case in practice, which has the time constraints on the computing time.

\section{Problem Formulations}

We have described the operational process in MPS in the first section of this paper. The garment assembly work which needs to be processed by the module is "tasks." Different tasks require different operations on them. The tasks can be classified into different "classes." The tasks having the same assembly requirements belong to the same "class." Figure 1 illustrates a typical operational process for a specific class of tasks in a module in MPS.

In this figure, the rectangle represents the module and the circles represent the module members at different operations. The module members are "servers." Each server performs one specific operation on the tasks. There is a buffer before each server. When a class $i$ task proceeds to Server $j$, it will get 


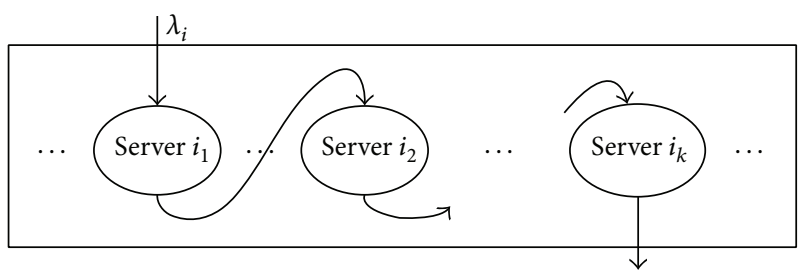

FIGURE 1: A typical operational process for a specific class of tasks in a module in MPS.

service (processed) immediately if the buffer before Server $j$ is empty and the Server $j$ is free; otherwise, it has to join the queue of class $i$ tasks in the buffer until all the class $i$ tasks before it in the queue get processed and Server $j$ chooses to work on a class $i$ task next. Only then, that class $i$ task begins processing. After getting processed by Server $j$, that class $i$ task moves to the buffer before the server which will perform the next operation on it and waits for getting processed. This process continues until all needed operations are done; then that class $i$ task exists the module.

From the queueing theory perspective, the module in which the tasks get processed is a queueing system with multiple classes of tasks and multiple servers. The arrival rate and the processing time for each class of tasks are stochastic. The route of each task is predetermined. Assume that each module member can perform all operations the task needed, and the performance of every module member on any operation is the same. The capacity of each server can be dynamically adjusted. How to dynamically allocate the module's capacity to each server so that the specific performances of interested are optimized is an optimal scheduling problem in this queueing system.

In the next two subsections, two mathematical models are constructed to achieve the optimal allocation of the module's capacity to each server. These models can be shown to be SCLP and SCCP and can be solved efficiently by using the methods we mentioned in Section 2.2. Before we proceed, we need to define some notations which we will use in the remaining of this paper.

\section{Notations}

(i) For tasks in class $i$, the number of the operations needed is denoted as $k_{i}$.

(ii) We use $\left\{i_{1}, \ldots, i_{k_{i}}\right\}$ to denote the order of operations for processing a class $i$ task if it is processed by Server $i_{1}$ firstly and then Sever $i_{2}$ until Sever $i_{k_{i}}$.

(iii) $\lambda_{i}(t)$ denotes the arrival rate of class $i$ tasks to the module at time $t, i=1, \ldots, n$.

(iv) $\mu_{i i_{j}}$ denotes the processing time of class $i$ tasks on Server $i_{j}, i=1, \ldots, n, j=1, \ldots, k_{i}$.

(v) $c_{i i_{j}}$ denotes the rate of holding cost of class $i$ tasks in the buffer before Server $i_{j}, i=1, \ldots, n, j=1, \ldots, k_{i}$.

(vi) $a_{i i_{j}}$ denotes the amount of class $i$ tasks in the buffer before Server $i_{j}$ at time 0 ; here $i=1, \ldots, n, j=$ $1, \ldots, k_{i}$.
3.1. SCLP Model. We consider the optimal allocation of the module's capacity within a time interval $[0, T]$. The objective is minimizing the holding cost of the WIP inventory in the module during $[0, T]$. The following is the model we construct.

Model 1:

$$
\begin{array}{cc}
\min \quad \sum_{i=1}^{n} \sum_{j=1}^{k_{i}}\left(\int_{0}^{T} c_{i i_{j}} x_{i i_{j}}(t)\right) d t & \\
\text { s.t. } \quad & x_{i i_{1}}(t)=a_{i i_{1}}+\int_{0}^{t} \lambda_{i}(s) d s-\int_{0}^{t} u_{i i_{1}}(s) d s, \\
& i=1, \ldots, n \\
x_{i i_{j}}(t)=a_{i i_{j}}+\int_{0}^{t} u_{i i_{j-1}}(s) d s-\int_{0}^{t} u_{i i_{j}}(s) d s, & \quad i=1, \ldots, n ; \quad j=1, \ldots, k_{i} ; \\
\sum_{i=1}^{n} \sum_{j=1}^{k_{i}}\left(\mu_{i i_{j}} u_{i i_{j}}(t)\right) \leq 1, \\
u_{i i_{j}}(t), x_{i i_{j}}(t) \geq 0, \quad i=1, \ldots, n, \\
j=1,2, \ldots, k_{i}, \quad t \in[0, T],
\end{array}
$$

where $x_{i i_{j}}(t)$ is the state variable and represents the amount of class $i$ tasks in the buffer before Server $i_{j}$ at time $t$ and $u_{i i_{j}}(t)$ is the control variable and represents the processing rate for class $i$ tasks on Server $i_{j}$ at time $t, i=1, \ldots, n, j=1,2, \ldots, k_{i}$.

The objective (4) is minimizing the total holding cost of WIP inventory in the module within the time interval $[0, T]$.

The constraints (5) and (6) describe the dynamic of the assembly process in the module, that is, how the state variables $x_{i i_{j}}(t)$ are changing with the changing of the control variables $u_{i i_{j}}(t)$.

The constraint (5) states that the amount of class $i$ tasks in the buffer before Server $i_{1}$ at any time $t$ is equal to the summation of the amount of class $i$ tasks in the buffer before Server $i_{1}$ at time 0 and the amount of class $i$ tasks arrived from outside during $[0, t]$ lessening the amount of class $i$ tasks processed by Server $i_{1}$ during $[0, t]$.

The constraint (6) states that the amount of class $i$ tasks in the buffer before Server $i_{j}$ at time $t$ is equal to the summation of the amount of class $i$ tasks in the buffer before Server $i_{j}$ at time 0 and the amount of class $i$ tasks processed by Server $i_{j-1}$ during $[0, t]$ lessening the amount of class $i$ tasks processed by Server $i_{j}$ during $[0, t], j=2, \ldots, k_{i}$.

The constraint (7) is the processing capacity constraints. It states that the total capacity consumed by the tasks on all the servers cannot exceed the module's capacity, which is 1 , at any time $t$.

Although the arrival rate of class $i$ task, $\lambda_{i}(t)$, is timevarying parameters in practice, the variation of it within a sufficient short time interval, for example, within one day, is small. Thus we can choose a sufficient small $T$ and replace $\lambda_{i}(t)$ with $\lambda_{i}$, when $t \in[0, T]$. With this simplification, Model 1 reduces to the following SCLP problem. 
Model 1':

$$
\begin{array}{cl}
\min & \sum_{i=1}^{n} \sum_{j=1}^{k_{i}}\left(\int_{0}^{T} c_{i i_{j}} x_{i i_{j}}(t)\right) d t \\
\text { s.t. } & \int_{0}^{t} u_{i i_{1}}(s) d s+x_{i i_{1}}(t)=a_{i i_{1}}+\lambda_{i} t, \quad i=1, \ldots, n \\
& \int_{0}^{t}\left[u_{i i_{j}}(s)-u_{i i_{j-1}}(s)\right] d s-x_{i i_{j}}(t)=a_{i i_{j}}, \\
& i=1, \ldots, n ; \quad j=1, \ldots, k_{i} ; \\
& \sum_{i=1}^{n} \sum_{j=1}^{k_{i}}\left(\mu_{i i_{j}} u_{i i_{j}}(t)\right) \leq 1, \quad i=1, \ldots, n, \\
u_{i i_{j}}(t), x_{i i_{j}}(t) \geq 0, \quad i=[0, T] . \\
j=1,2, \ldots, k_{i}, \quad t
\end{array}
$$

We can use algorithm described in Section 2.2 to solve Model 1' efficiently.

3.2. SCCP Model. Suppose every buffer has its target inventory level for WIP inventory. For the buffer before Server $i_{j}$, the target level is denoted as $d_{i i_{j}}$. The following model can be used to find the optimal allocation of the module's capacity with the objective of minimizing the total deviation of the WIP inventories from their corresponding target levels in the module during $[0, T]$.

Model 2:

$$
\begin{gathered}
\min \quad \int_{0}^{T} \sum_{i=1}^{n} \sum_{j=1}^{k_{i}}\left[\left(x_{i i_{j}}(t)-d_{i_{j}}\right)^{2}\right] d t \\
\text { s.t } \quad x_{i i_{1}}(t)=a_{i i_{1}}+\int_{0}^{t} \lambda_{i}(s) d s-\int_{0}^{t} u_{i i_{1}}(s) d s, \\
\quad i=1, \ldots, n \\
x_{i i_{j}}(t)=a_{i i_{j}}+\int_{0}^{t} u_{i i_{j-1}}(s) d s-\int_{0}^{t} u_{i i_{j}}(s) d s, \quad j=1, \ldots, k_{i} ; \\
i=1, \ldots, n ; \quad j=1, \ldots, n, \\
\sum_{i=1}^{n} \sum_{j=1}^{k_{i}}\left(\mu_{i i_{j}} u_{i i_{j}}(t)\right) \leq 1, \\
\left.u_{i i_{j}}(t), x_{i i_{j}}(t) \geq 0, \quad i=1, \ldots, T\right] . \\
j=i_{1}, i_{2}, \ldots, k_{i}, \quad t \in[0, T]
\end{gathered}
$$

The objective (9) is minimizing the total deviation of the WIP inventories from their corresponding target levels in all the buffers.

The remaining constraints in Model 2 are the same as those in Model 1.

Model 2 can be written into the SCCP form (2) by using the variables transformation. We omit the details of the variables transformation and interested reader can refer to section 5.2 in [40].

We can use the algorithm mentioned in Section 2.2 to solve Model 2 efficiently.

3.3. Implementation of the Solutions for Models 1 and 2. In the above two subsections, we propose the models to find the optimal allocation of a module's capacity to different operations for given assembly tasks so that the holding cost of the WIP inventory and the total deviation of the WIP inventories from their corresponding target levels in the module during $[0, T]$ are minimized.

After we solve the two models, we get $u_{i i_{j}}(t)$ which represents the processing rate for class $i$ tasks on Server $i_{j}$, where $i=1, \ldots, n, j=i_{1}, i_{2}, \ldots, k_{i}, t \in[0, T]$. We can allocate the module members to different operations according to the value of $u_{i i_{j}}(t)$. The number of module members in a specific operation, for example, the $i_{j}$ th operation, is $u_{i i_{j}} \times$ the total number of module members in the module.

If every module member only can perform several (not all) operations and/or the efficiency for performing the same operation is different for different module members, the capacity of each module member can be represented by a vector and each component of the vector represents the capacity of the module member on performing a specific operation. Each server's capacity is the summation of capacity of each module member on performing the operation the server represents. The module's capacity constraint in Models 1 and 2 then should be replaced by capacity constraints for each server and each module member.

When the values of some capacities change, for example, if one module member becomes ill and cannot perform any operations, the total capacity of the module also changes. The models can be recomputed and the new allocation solutions can be got quickly.

\section{Conclusion and Future Work}

In this paper, we construct two mathematical models to find the optimal allocation of the module's capacity to the operations for given tasks, under the assumption that each module member can perform all operations the task needed, and the performance of every module member on any operation is the same. The objectives are minimizing the holding cost for WIP inventory in the module and the total deviation of the WIP inventories from their corresponding target values during a specific time interval. The solutions of the models can be used as reference to achieve better allocation of the module members to operations for given tasks.

We also discuss the modifications of the models when each module member only can perform several (not all) operations, and/or the efficiency for performing the same operation is different for different module members.

To our knowledge, this is the only paper in the literature focusing on the implementation of MPS on the operational level by addressing the optimal allocation of module members to operations in a module. 
There are some research directions needing to be pursued in the future. For example, how to use the result of this paper to derive the training plan for the module member so that the total capacity of a module can be increased greatly in the short and/or long term is an important issue for facilitating the adoption of MPS.

\section{Conflict of Interests}

The authors declare that there is no conflict of interests regarding the publication of this paper.

\section{Acknowledgments}

This work is supported in part by the Natural Science Foundation of China under Grant 71371197 and the 985 Project of Sun Yat-Sen University.

\section{References}

[1] M. Christopher, "The agile supply chain: competing in volatile markets," Industrial Marketing Management, vol. 29, no. 1, pp. 37-44, 2000.

[2] T.-M. Choi and S. Sethi, "Innovative quick response programs: a review," International Journal of Production Economics, vol. 127, no. 1, pp. 1-12, 2010.

[3] A. Şen, "The US fashion industry: a supply chain review," International Journal of Production Economics, vol. 114, no. 2, pp. 571-593, 2008.

[4] M. Bruce, L. Daly, and N. Towers, "Lean or agile: a solution for supply chain management in the textiles and clothing industry?" International Journal of Operations \& Production Management, vol. 24, no. 1-2, pp. 151-170, 2004.

[5] G. L. Hodge, K. Goforth Ross, J. A. Joines, and K. Thoney, "Adapting lean manufacturing principles to the textile industry," Production Planning and Control, vol. 22, no. 3, pp. 237-247, 2011.

[6] M. Bruce and L. Daly, "Adding value: challenges for UK apparel supply chain management-a review," Production Planning and Control, vol. 22, no. 3, pp. 210-220, 2011.

[7] F. Caro and J. Gallien, "Inventory management of a fast-fashion retail network," Operations Research, vol. 58, no. 2, pp. 257-273, 2010.

[8] Z. L. Chen, "Integrated production and outbound distribution scheduling: review and extensions," Operations Research, vol. 58, no. 1, pp. 130-148, 2010.

[9] I. M. Taplin, "Backwards into the future: new technologies and old work organization in the US clothing industry," in Rethinking Global Production, I. M. Taplin and J. Winterton, Eds., Ashgate, Brookfield, Vt, USA, 1997.

[10] Z. X. Guo, W. K. Wong, S. Y. S. Leung, J. T. Fan, and S. F. Chan, "Mathematical model and genetic optimization for the job shop scheduling problem in a mixed- and multi-product assembly environment: a case study based on the apparel industry," Computers and Industrial Engineering, vol. 50, no. 3, pp. 202219, 2006

[11] T.-M. Choi, N. Liu, S. Ren, and C. Hui, "No refund or full refund: when should a fashion brand offer full refund consumer return service for mass customization products?" Mathematical Problems in Engineering, vol. 2013, Article ID 561846, 14 pages, 2013.
[12] Y. He and J. Zhang, "Random yield risk sharing in a two-level supply chain," International Journal of Production Economics, vol. 112, no. 2, pp. 769-781, 2008.

[13] H. Peng and M. Zhou, "Quantity discount supply chain models with fashion products and uncertain yields," Mathematical Problems in Engineering, vol. 2013, Article ID 895784, 11 pages, 2013.

[14] B. Dong, H. Jia, Z. Li, and K. Dong, "Implementing mass customization in garment industry," Systems Engineering Procedia, vol. 3, pp. 372-380, 2012.

[15] R. Huang and S. Yu, "Clarifying cutting and sewing processes with due windows using an effective ant colony optimization," Mathematical Problems in Engineering, vol. 2013, Article ID 182598, 12 pages, 2013.

[16] M. C. Gomes, A. P. Barbosa-Póvoa, and A. Q. Novais, "Optimal scheduling for flexible job shop operation," International Journal of Production Research, vol. 43, no. 11, pp. 2323-2353, 2005.

[17] C. Y. Baldwin and K. B. Clark, "Managing in an age of modularity," Harvard Business Review, vol. 75, no. 5, pp. 84-93, 1997.

[18] A. J. Chuter, Introduction to Clothing Production Management, BSP Professional Books, Oxford, UK, 1988.

[19] E. Hill, Flexible Work Groups, Clemson Apparel Research, Clemson University, Clemson, SC, USA, 1994.

[20] J. T. Dunlop and D. Weil, "Diffusion and performance of modular production in the U.S. apparel industry," Industrial Relations, vol. 35, no. 3, pp. 334-355, 1996.

[21] P. Berg, E. Appelbaum, T. Bailey, and A. L. Kalleberg, "The performance effects of modular production in the apparel industry," Industrial Relations, vol. 35, no. 3, pp. 356-373, 1996.

[22] F. H. Abernathy, J. T. Dunlop, J. H. Hammond, and D. Weil, "The information integrated channel: a study of the US apparel industry in transition," in Brookings Papers on Economic Activity: Microeconomics, pp. 175-246, 1995.

[23] J. D. Blackburn, "The quick response movement in the apparel industry: a case study in time-compressing supply chains," in Time-Based Competition, J. D. Blackburn, Ed., Business One/Irwin, Homewood, Ill, USA, 1991.

[24] T. Bailey, P. Berg, and C. Sandy, "The effect of high-performance work practices on employee earnings in the steel, apparel, and medical electronics and imaging industries," Industrial and Labor Relations Review, vol. 54, no. 2A, pp. 525-543, 2001.

[25] W. A. S. Castro, R. C. Castro, S. I. Mirón, and P. U. Alonso Martínez, "Modular manufacturing: an alternative to improve the competitiveness in the clothing industry," International Journal of Clothing Science and Technology, vol. 16, no. 3-4, pp. 301-309, 2004.

[26] S. X. Xu, Q. Lu, and Z. Li, "Optimal modular production strategies under market uncertainty: a real options perspective," International Journal of Production Economics, vol. 139, no. 1, pp. 266-274, 2012.

[27] C. Forza and A. Vinelli, "Time compression in production and distribution within the textile-apparel chain," Integrated Manufacturing Systems, vol. 11, no. 2, pp. 138-146, 2000.

[28] S. Lin, M. A. Moore, D. H. Kincade, and C. Avery, "Dimensions of apparel manufacturing strategy and production management," International Journal of Clothing Science and Technology, vol. 14, no. 1, pp. 46-60, 2002.

[29] R. N. Tomastik, P. B. Luh, and G. Liu, "Scheduling flexible manufacturing systems for apparel production," IEEE Transactions on Robotics and Automation, vol. 12, no. 5, pp. 789-799, 1996. 
[30] D. M. Rose and D. R. Shier, "Cut scheduling in the apparel industry," Computers \& Operations Research, vol. 34, no. 11, pp. 3209-3228, 2007.

[31] H. M. Hsu, Y. Hsiung, Y. Z. Chen, and M. Wu, "A GA methodology for the scheduling of yarn-dyed textile production," Expert Systems with Applications, vol. 36, no. 10, pp. 12095-12103, 2009.

[32] W.-K. Yeung, T.-M. Choi, and T. C. E. Cheng, "Optimal scheduling of a single-supplier single-manufacturer supply chain with common due windows," Institute of Electrical and Electronics Engineers. Transactions on Automatic Control, vol. 55, no. 12, pp. 2767-2777, 2010.

[33] W.-K. Yeung, T.-M. Choi, and T. C. E. Cheng, "Supply chain scheduling and coordination with dual delivery modes and inventory storage cost," International Journal of Production Economics, vol. 132, no. 2, pp. 223-229, 2011.

[34] T. Choi, W. Yeung, and T. C. E. Cheng, "Scheduling and co-ordination of multi-suppliers single-warehouse-operator single-manufacturer supply chains with variable production rates and storage costs," International Journal of Production Research, vol. 51, no. 9, pp. 2593-2601, 2013.

[35] D. G. Luenberger and Y. Ye, Linear and Nonlinear Programming, vol. 116 of International Series in Operations Research \& Management Science, Springer, New York, NY, USA, 3rd edition, 2008.

[36] E. J. Anderson, A continuous model for job-shop scheduling [Ph.D. thesis], University of Cambridge, Cambridge, UK, 1978.

[37] G. Weiss, "A simplex based algorithm to solve separated continuous linear programs," Mathematical Programming A, vol. 115, no. 1, pp. 151-198, 2008.

[38] E. J. Anderson and P. Nash, Linear Programming in InfiniteDimensional Spaces: Theory and Applications, John Wiley \& Sons, Chichester, UK, 1987.

[39] X. Wang, "Review on the research for separated continuous linear programming: with applications on service operations," Mathematical Problems in Engineering, vol. 2013, Article ID 408048, 9 pages, 2013.

[40] X. Wang, S. Zhang, and D. D. Yao, "Separated continuous conic programming: strong duality and an approximation algorithm," SIAM Journal on Control and Optimization, vol. 48, no. 4, pp. 2118-2138, 2009.

[41] Y. Nesterov and A. Nemirovskii, Interior-Point Polynomial Algorithms in Convex Program Ming, SIAM, Philadelphia, $\mathrm{Pa}$, USA, 1994. 




Advances in

Operations Research

mansans

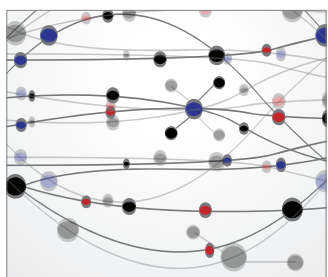

The Scientific World Journal
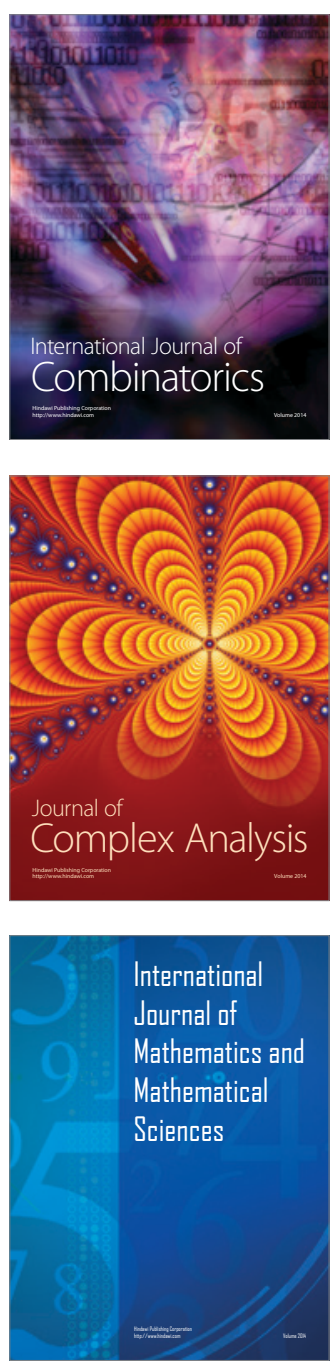
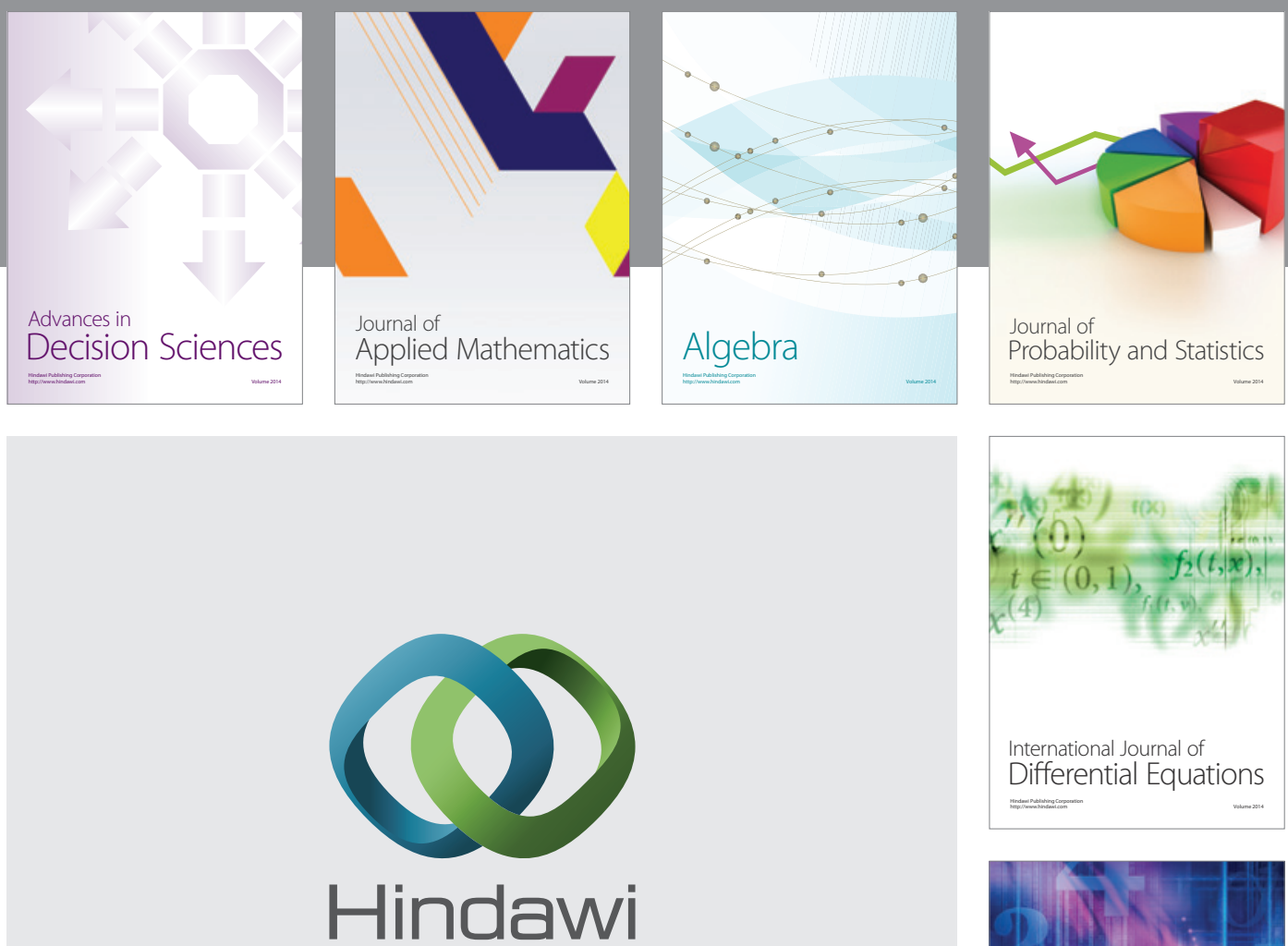

Submit your manuscripts at http://www.hindawi.com
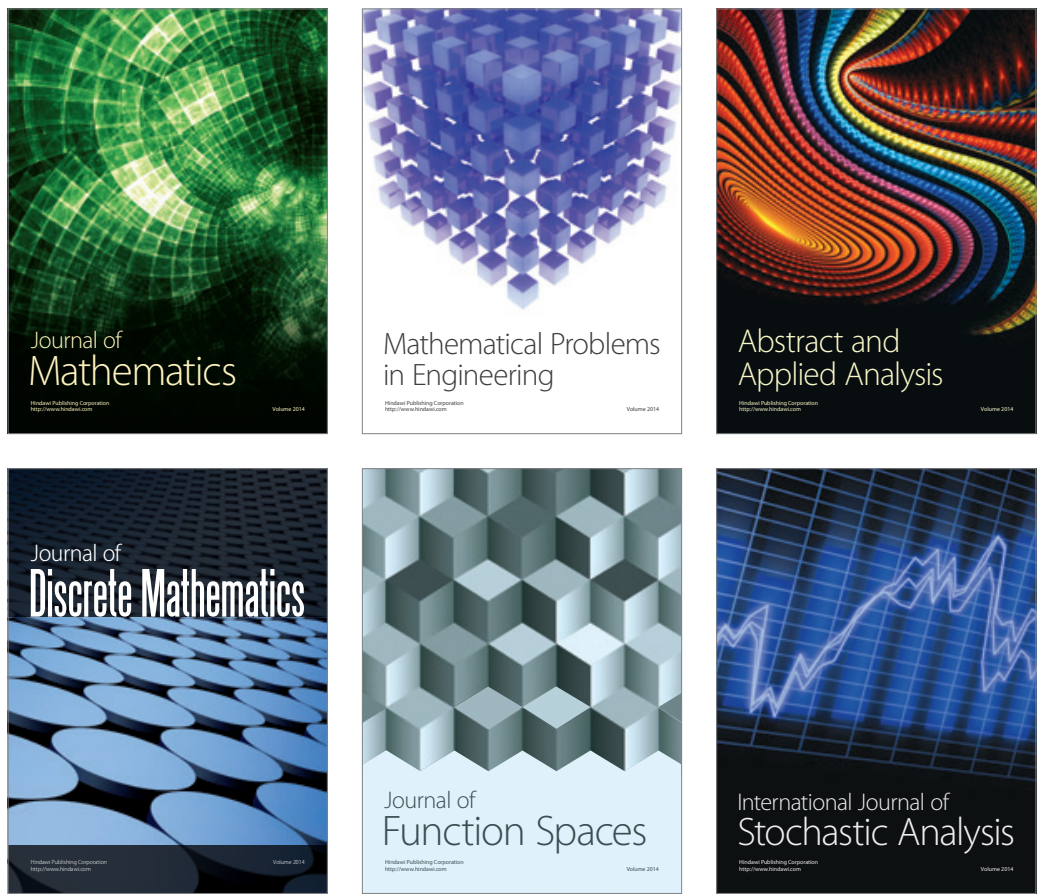

Journal of

Function Spaces

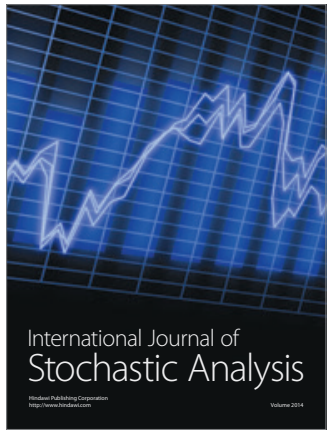

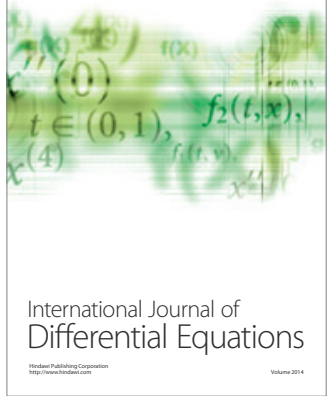
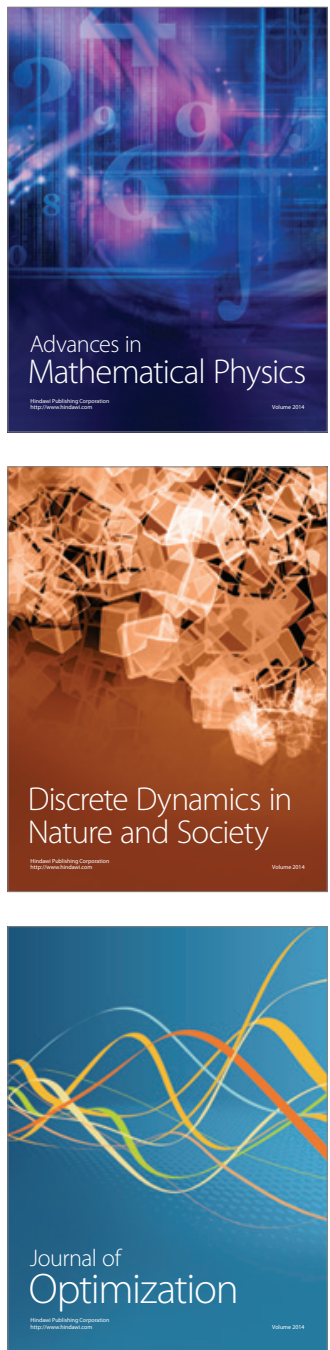